\title{
ON LERAY'S SELF-SIMILAR SOLUTIONS OF THE NAVIER-STOKES EQUATIONS SATISFYING LOCAL ENERGY ESTIMATES
}

\author{
TAI-PENG TSAI
}

\begin{abstract}
This paper proves that Leray's self-similar solutions of the threedimensional Navier-Stokes equations must be trivial under very general assumptions, for example, if they satisfy local energy estimates.
\end{abstract}

\section{INTRODUCTION}

In 1934 Leray [Le] raised the question of the existence of self-similar solutions of the Navier-Stokes equations. For a long time, the self-similar solutions had appeared to be a good candidate for constructing singular solutions of the Navier-Stokes equations. Leray's question was unanswered until 1995, when Nečas, Růžička, and Šverák [NRS] showed, among other things, that the only self-similar solution satisfying the global energy estimates is zero. Although they answered Leray's original problem, some important questions were left open. For example, can a selfsimilar solution satisfying local energy estimates exist? The goal of this paper is to show that the self-similar solutions must be zero under very general assumptions, for example, if they satisfy the local energy estimates.

For the Navier-Stokes equations

$$
\begin{aligned}
u_{t}-\nu \Delta u+u \cdot \nabla u+\nabla p & =0 \\
\operatorname{div} u & =0
\end{aligned} \quad \text { in } \mathbf{R}^{3} \times\left(t_{1}, t_{2}\right)
$$

with $\nu>0$, Leray's (backward) self-similar solutions are of the form

$$
\begin{aligned}
& u(x, t)=\lambda(t) U(\lambda(t) x) \\
& p(x, t)=\lambda^{2}(t) P(\lambda(t) x)
\end{aligned} \quad \text { with } \lambda(t)=\frac{1}{\sqrt{2 a(T-t)}},
$$

where $a>0, U(y)=\left(U_{1}, U_{2}, U_{3}\right)(y)$ and $P(y)$ are defined in $\mathbf{R}^{3}$. One also requires that certain natural energy norms of $u$ be finite. (Otherwise there exist nontrivial solutions. See Remark 5.4.) The Navier-Stokes equations for $u$ give the system

$$
\begin{aligned}
-\nu \Delta U+a U+a(y \cdot \nabla) U & +(U \cdot \nabla) U+\nabla P=0 \quad \text { in } \mathbf{R}^{3} \\
\operatorname{div} U & =0
\end{aligned}
$$

Date. January 8, 1997. 
for $U$. As suggested by Leray, a nonzero $U$ would produce a solution $u$ of (1.1) with a singularity at $(0, T)$. This would give a counterexample to the open question whether a solution of (1.1) satisfying natural energy estimates can develop a singularity.

In addition to looking for particular singular solutions, the study of self-similar solutions seems to be important also from a more general point of view. It is related to the scaling property of (1.1), the fact that if $u(x, t)$ solves $(1.1)$, then so do the rescaled functions

$$
u_{r}(x, t):=r u\left(r x, r^{2}(t-T)+T\right)
$$

for each $r>0$. If $(0, T)$ is a singular point, then the asymptotics of the singularity is encoded in the behavior of $u_{r}$ as $r \rightarrow 0^{+}$. If $u_{r}$ converges to a limit $\bar{u}$, the limit $\bar{u}$ must be self-similar, i.e. $(\bar{u})_{r}=\bar{u}$ for all $r>0$, which implies $\bar{u}$ is of the form (1.2). Of course, more complicated singularities may possibly exist. The study of self-similar solutions has proved to be very useful in the investigations of singularities of many equations with similar scaling properties, such as the harmonic map heat flow, semilinear heat equations, and nonlinear Schrödinger equations, see for example [Str], [GK], [KL]. It is hoped that the study of (1.3) can shed some light on the regularity question for the Navier-Stokes equations.

The known regularity criteria for Navier-Stokes equations (such as [Se], [FJR], [vW, p190], [Gi], [Str2], [Ta], [CF]) do not apply to self-similar singularities (unless certain quantities are small). The main result of [NRS] is that the only weak solution of (1.3) belonging to $L^{3}\left(\mathbf{R}^{3}\right)$ is $U \equiv 0$. Also see [MNPS]. (They showed the same conclusion under a stronger assumption, but without using results from [CKN].) The $L^{3}$ integrability condition will hold if the corresponding solution $u$ of the Navier-Stokes equations satisfies the global energy estimates

$$
\int_{\mathbf{R}^{3}} \frac{1}{2}|u(x, t)|^{2} d x+\int_{t_{1}}^{t} \int_{\mathbf{R}^{3}} \nu|\nabla u(x, t)|^{2} d x d t \leq \int_{\mathbf{R}^{3}} \frac{1}{2}\left|u\left(x, t_{1}\right)\right|^{2} d x
$$

for all $t \in\left(t_{1}, t_{2}\right)$. On the other hand, if one only assumes the local energy estimates,

$$
\text { ess } \sup _{t_{3}<t<T} \int_{B} \frac{1}{2}|u(x, t)|^{2} d x+\int_{t_{3}}^{T} \int_{B} \nu|\nabla u(x, t)|^{2} d x d t<\infty
$$

for some ball $B$ and some $t_{3}<T$, one only gets estimates of some weighted norms which do not imply $U \in L^{3}$. (See Section 4 for more details.) Therefore, [NRS] left open the existence of self-similar singularities which satisfy the local energy estimates. For example, a solution with the following decay was not excluded:

$$
U(y)=A\left(\frac{y}{|y|}\right) \frac{1}{|y|}+o\left(\frac{1}{|y|}\right) \quad \text { as } y \rightarrow \infty,
$$

where $A: S^{2} \rightarrow \mathbf{R}^{3}$ is smooth. At the same time, this seems to be a very natural candidate for a self-similar singularity: the function $u$ given by $(1.2)_{1}$ will satisfy the local energy estimates, and $u(x, t) \rightarrow u_{T}(x)$ as $t \rightarrow T^{-}$, where $u_{T}(x)=A(x /|x|) /|x|$ is homogeneous of degree -1 . One might speculate that after the blow-up time $T u$ would become a forward (or de-focusing) self-similar solution, (the existence of such solutions was studied in $[\mathrm{GM}],[\mathrm{CP}]$ ), providing a rather nice interior singularity. In addition, as suggested in [CKN], the blowup rate of a singularity of $u$ at $(0, T)$ 
is (at least in "parabolic average")

$$
|u(x, t)| \geq \frac{C}{|x|+\sqrt{T-t}}
$$

which is satisfied by a solution $u$ given by (1.2) if $U$ has the decay (1.5).

In this paper we exclude the possibility of such self-similar singularities. In fact, we prove the following results.

Theorem 1. If a weak solution $U$ of (1.3) belongs to $L^{q}\left(\mathbf{R}^{3}\right)$, for some $q \in(3, \infty]$, then it must be constant (and hence identically zero if $q<\infty$ ).

Theorem 2. Suppose $u$ is a weak solution of (1.1) satisfying the local energy estimates (1.4) in the cylinder $Q_{1}(0, T)=B_{1}(0) \times(T-1, T)$. If $u$ is of the form (1.2) $)_{1}$, then $u$ is identically zero.

We refer the reader to Section 2 for the definitions of weak solutions. A particular corollary of these results is that a weak solution $U$ of (1.3) with the decay (1.5) must be zero.

Let us explain the main idea of the proof. We recall from [NRS] that the smooth function

$$
\Pi(y)=\frac{1}{2}|U(y)|^{2}+P(y)+a y \cdot U(y)
$$

satisfies

$$
-\nu \Delta \Pi(y)+(U(y)+a y) \cdot \nabla \Pi(y)=-\nu|\Omega(y)|^{2} \leq 0,
$$

where $\Omega=$ curl $U$. (Hence $\Pi$ satisfies the maximal principle.) One of the crucial steps in [NRS] was to show

$$
\Pi(y)=o(1) \quad \text { as } y \rightarrow \infty .
$$

They achieved this by showing

$$
U(y)=O\left(|y|^{-3}\right), \quad P(y)=O\left(|y|^{-2}\right) \quad \text { as } y \rightarrow \infty
$$

under the assumption $U \in L^{3}\left(\mathbf{R}^{3}\right)$. Using their method in the case $U \in L^{q}\left(\mathbf{R}^{3}\right)$, $3<q<9$, one only gets weaker estimates

$$
U(y)=O\left(|y|^{-1}\right), \quad P(y)=O\left(|y|^{-2+\sigma}\right) \quad \text { as } y \rightarrow \infty
$$

for arbitrary small $\sigma>0$, from which (1.8) does not follow. This seems to be a serious obstacle for generalizing the method used in [NRS] to $q>3$.

Our key observation is that (1.9) and, in fact, even a much weaker condition

$$
U(y)=o(|y|), \quad P(y)=O\left(|y|^{N}\right) \quad \text { as } y \rightarrow \infty
$$

for some finite $N$ (no matter how large), is sufficient to imply $\Pi$ is constant. Heuristically, for the differential equation

$$
\Delta v(y)=y \cdot \nabla v(y)
$$

the right hand side is a "magnifying force", (cf. 1-D case: $\left.v^{\prime \prime}=x v^{\prime}\right)$. Therefore, a solution should either be constant, or grow unboundedly. In addition, in $R^{3}$, a radial solution $v(y)=\phi(r), r=|y|$, satisfies

$$
\phi^{\prime}(r)=c \cdot \frac{1}{r^{2}} \exp \left(\frac{r^{2}}{2}\right)
$$


which suggests that a nonconstant solution $\Pi$ blows up at the same rate as $\phi$. This also suggests that we can find suitable comparison functions with very fast growth. Based on this observation, we will prove a Liouville type lemma which implies $\Pi$ is constant under the assumption (1.10) in Section 5. In the other part of this paper, we establish the estimates (1.10).

It should be emphasized that Theorem 2 is purely local in the sense that we do not impose any boundary condition on $u$. This is related to one special aspect of our analysis. In the study of Navier-Stokes equations, the pressure is usually considered as a "global" term and can be difficult to deal with. See for example [CKN], [SvW], [Str2], [LL], [HW]. When there is no boundary assumption on $u$, in general one can not obtain a "desired" estimate of $p$ for every weak solution $u$. In our analysis, we overcome this difficulty by making use of the self-similarity: the local estimate of $p$ corresponds to a global estimate of $P$, which we obtain by applying certain results on singular integrals on the space $B M O$ and on some weighted spaces with the weights in the class $A_{p}$. In particular, we are able to show that every self-similar weak solution $u$ in Theorem 2 is a "suitable weak solution" in the sense of [CKN], and then apply the partial regularity result in $[\mathrm{CKN}]$ to obtain (1.10).

Our plan for this paper is as follows. In Section 2 we recall the definitions and prove some results about the pressure. In Section 3 we prove the growth estimates (1.10) for Theorem 1, using the representation formula for Stokes system and certain results from harmonic analysis. In Section 4 we prove the estimates (1.10) for Theorem 2, using $A_{p}$ weights and a variant of Proposition 2 from [CKN]. In Section 5 we prove the Liouville type lemma and conclude Theorem 1 and Theorem 2 . To understand quickly the main idea, the reader may just assume (1.10) and go directly to Section 5 .

\section{Preliminaries}

This section establishes notational conventions and some definitions. First we discuss the notation. We use $Q_{r}$ to denote the parabolic cylinders

$$
Q_{r}(x, T)=B_{r}(x) \times\left(T-r^{2}, T\right) .
$$

We will also use the summation convention. We write $u_{i, j}=\partial_{j} u_{i}=\frac{\partial u_{i}}{\partial x_{j}}$, and use the letters $C$ and $c$ to denote generic constants which may change from line to line.

As far as the definitions of solutions of the Navier-Stokes equations (1.1) are concerned, we refer the reader to [CKN] for the concept of Leray-Hopf weak solutions, ([Le], $[\mathrm{Ho}])$, and the concept of suitable weak solutions, ([Sch1], [CKN]). Also see $[\mathrm{CFo}],[\mathrm{Te}],[\mathrm{vW}]$. We recall that the main ingredients of the definition of suitable weak solutions in parabolic cylinders $Q_{r}$ are:

(i) the equation (1.1), interpreted in the sense of distributions,

(ii) the local energy estimates (1.4),

(iii) the assumption that the pressure $p$ belong to $L^{5 / 4}\left(Q_{r}\right)$, and

(iv) the generalized energy inequality, (see [CKN] p.779).

When we consider Leray-Hopf weak solutions, only (i), (ii) and an energy inequality (instead of (iv)) are required. Among the above conditions, (iv) is satisfied by any smooth solution of (1.1). Also, (iii) can be derived from (i), (ii), and (iv) if we impose a 0 -boundary condition on $u$. See [SvW], [LL]. However, in general (iii) is 
not a result of (i), (ii), and (iv), as can be seen from the following example. We use Serrin's idea ([Se], p.187) and consider the vector field $u$ defined by

$$
u(x, t)=(T-t)^{s}(1,0,0), \quad 0<s<\frac{1}{5} .
$$

$u$ is a Leray-Hopf weak solution in $Q_{1}(0, T)$ (with non-homogeneous boundary condition). (In fact, $u$ is a Leray-Hopf weak solution in $B_{1}(0) \times(T-1, T+1)$ if we extend $u$ to $B_{1}(0) \times(T, T+1)$ by zero.) However, since

$$
p(x, t)=s(T-t)^{s-1} x_{1}+\text { const., }
$$

$p$ does not belong to $L^{5 / 4}\left(Q_{1}\right)$ and hence $(u, p)$ is not a suitable weak solution in $Q_{1}$. We remark that, in Theorem 2, we do not require the weak solution $u$ to be a Leray-Hopf weak solution. Our only requirements (apart from self-similarity) are (i) and (ii): the Navier-Stokes equations and the local energy estimates.

A function $U$ is called a weak solution of (1.3) if $U=\left(U_{1}, U_{2}, U_{3}\right) \in W_{l o c}^{1,2}\left(\mathbf{R}^{3}\right)$, $\operatorname{div} U=0$, and

$$
\int_{\mathbf{R}^{3}}(\nu \nabla U \cdot \nabla \varphi+[a U+a(y \cdot \nabla) U+(U \cdot \nabla) U] \cdot \varphi) d x=0
$$

for all $\varphi=\left(\varphi_{1}, \varphi_{2}, \varphi_{3}\right) \in C_{c}^{\infty}\left(\mathbf{R}^{3}\right)$, div $\varphi=0$. By standard regularity theory of stationary Navier-Stokes equations, every weak solution $U$ of (1.3) is actually smooth, (see for example [Ga II], [GiM], [La], [Te]).

Regarding the pressure, in the definition of a weak solution $U$, we do not require the specification of the pressure function. On the other hand, given a weak solution $U$, one can always locally define $P$ such that $(U, P)$ solves (1.3) in bounded regions. (For example, we can apply the results for Stokes system from [Ga I] p.180, with the body force $f=a U+a(y \cdot \nabla) U+(U \cdot \nabla) U$.) In any given connected region $P$ is unique up to a constant. Therefore we can define a pressure $P_{R}$ in each ball $B_{R}(0)$ and make them agree with each other by specifying $\int_{B_{1}} P_{R}=0$ (or a fixed constant). In this way we can define $P$ globally. Since $U$ is smooth, $P$ is also smooth.

Another way to define $P$ is by considering its equation. One takes the divergence of (1.3) and formally deduces

$$
-\Delta P=\sum \partial_{i} \partial_{j}\left(U_{i} U_{j}\right)
$$

If $2<q<\infty$, one can define

$$
\widetilde{P}=\sum R_{i} R_{j}\left(U_{i} U_{j}\right)
$$

(cf. [NRS]), where $R_{j}, j=1,2,3$, are the classical Riesz transforms, (see for example [St]). We will show that $\widetilde{P}$ is smooth and differs from $P$ by a constant. We can also define $\widetilde{P}$ by $(2.2)$ for the case $q=\infty$. In that case, $\widetilde{P}$ is to be understood as a $B M O$ function and is defined by duality, see [JN], [FS], [St2, p.156]. We are interested in $\widetilde{P}$ since we can get a global control of $\widetilde{P}$ which will give us the desired local control of $P$. For clarification, we formulate the following lemma.

Lemma 2.1. Let a weak solution $U$ of (1.3) belong to $L^{q}\left(\mathbf{R}^{3}\right), 2<q \leq \infty$. Let $\widetilde{P}$ be defined by (2.2). Then $\widetilde{P}$ solves (2.1) in distributional sense, and hence is 
smooth. Moreover, we have

$$
\begin{array}{ll}
\|\widetilde{P}\|_{q / 2} \leq C\|U\|_{q}^{2} & \text { if } 2<q<\infty, \\
\|\widetilde{P}\|_{B M O} \leq C\|U\|_{\infty}^{2} & \text { if } q=\infty .
\end{array}
$$

Finally, if $P$ is defined as earlier in this section, then $P-\widetilde{P}$ is constant if $2<q<\infty$, and affine if $q=\infty$.

Proof. To show $\widetilde{P}$ is a distributional solution of (2.1), one has to show

$$
-\int \widetilde{P} \Delta \varphi=-\int R_{i} R_{j}\left(U_{i} U_{j}\right) \Delta \varphi=\int U_{i} U_{j} \partial_{i} \partial_{j} \varphi
$$

for all $\varphi \in C_{c}^{\infty}\left(\mathbf{R}^{3}\right)$. We recall that $R_{i} R_{j}$ are self-adjoint in $L^{2}\left(\mathbf{R}^{3}\right)$, (this can be checked easily by Fourier transform, cf. [St] p.58), and we have

$$
R_{i} R_{j} \Delta \varphi=-\partial_{i} \partial_{j} \varphi
$$

(see [St] p.59). Therefore, for the case $2<q<\infty$, (2.5) can be established for $U \in C_{c}^{\infty} \subset L^{2}$. One then extends this result to general $U \in L^{q}$ by approximation. For the case $q=\infty$, we first observe that all integrals in (2.5) converge absolutely since $\varphi$ has compact support and $\widetilde{P} \in B M O \subset L_{l o c}^{r}$ for all $r<\infty$. We next observe $\Delta \varphi$ is in the Hardy space $\mathcal{H}^{1}\left(\mathbf{R}^{3}\right)$ (since $\Delta \varphi \in C_{c}^{\infty}$ and has mean value zero) and, since $\widetilde{P}$ is defined by duality, (see [St2] p.156), we get

$$
-\int \widetilde{P} \Delta \varphi=-\int U_{i} U_{j}\left(R_{i} R_{j} \Delta \varphi\right)=\int U_{i} U_{j} \partial_{i} \partial_{j} \varphi
$$

This shows that $\widetilde{P}$ is a distributional solution of $(2.1)$.

We remark that, by definition and the fact that $\Delta \varphi \in C_{c}^{\infty}$ with mean value zero, $R_{i} R_{j} \Delta \varphi$ remains the same independently of whether $\Delta \varphi$ is considered as an $L^{2}$ function or as an $\mathcal{H}^{1}$ function.

Since $U$ is smooth and $\widetilde{P}$ solves (2.1) in distributional sense, $\widetilde{P}$ is also smooth by Weyl's lemma. The estimate (2.3) now follows from [CZ]. (2.4) is due to Spanne, Peetre, and Stein, (see [St2, p.191] for references). See also [St], [St2].

Finally, we show the last assertion by modifying the argument of [NRS], Lemma 3.1. Let

$$
F=-\nu \Delta U+a U+a(y \cdot \nabla) U+(U \cdot \nabla) U+\nabla \widetilde{P} .
$$

We know $F=\nabla \widetilde{P}-\nabla P$, hence $\Delta F=0$ in $\mathbf{R}^{3}$ since both $P$ and $\widetilde{P}$ solve (2.1). Therefore $F$ is analytic. We now claim that we have $D^{\alpha} F(0)=0$ for each $\alpha$ in the case $2<q<\infty$, and for each $\alpha$ with $|\alpha| \geq 1$ in the case $q=\infty$. (Clearly this is enough to conclude that $F$ is 0 (respectively, constant) by its analyticity.) To prove the claim, we note that, since $\Delta D^{\alpha} F=0$, for every radial function $\varphi \in C_{c}^{\infty}$ with $\int \varphi=1$, we have

$$
D^{\alpha} F(0)=(-1)^{|\alpha|} \int_{\mathbf{R}^{3}} F(y) \epsilon^{3+|\alpha|}\left(D^{\alpha} \varphi\right)(\epsilon y) d y
$$

([St, p.275]). We claim that, as $\epsilon \rightarrow 0$, all terms obtained by substituting (2.6) into the above integral converge to zero. Since the proof is similar to that in [NRS], we will only give an illustration and show how to deal with the terms involving $(y \cdot \nabla) U$ and $\nabla \widetilde{P}$ in the case $q=\infty$. 
For the term involving $(y \cdot \nabla) U$,

$$
\begin{aligned}
& \left|\int_{\mathbf{R}^{3}}(y \cdot \nabla) U \epsilon^{3+|\alpha|}\left(D^{\alpha} \varphi\right)(\epsilon y) d y\right| \\
= & \left|-\epsilon^{3+|\alpha|} \int_{\mathbf{R}^{3}} 3 U\left(D^{\alpha} \varphi\right)(\epsilon y) d y-\epsilon^{3+|\alpha|} \int_{\mathbf{R}^{3}} U \epsilon y_{j}\left(\partial_{j} D^{\alpha} \varphi\right)(\epsilon y) d y\right| \\
\leq & 3 \epsilon^{3+|\alpha|}\|U\|_{\infty}\left(\int_{\mathbf{R}^{3}}\left|\left(D^{\alpha} \varphi\right)(\epsilon y)\right| d y+\int_{\mathbf{R}^{3}}\left|\epsilon y_{j}\left(\partial_{j} D^{\alpha} \varphi\right)(\epsilon y)\right| d y\right) \\
= & 3 \epsilon^{3+|\alpha|}\|U\|_{\infty}\left(\int_{\mathbf{R}^{3}}\left|\left(D^{\alpha} \varphi\right)(z)\right| \epsilon^{-3} d z+\int_{\mathbf{R}^{3}}\left|z_{j}\left(\partial_{j} D^{\alpha} \varphi\right)(z)\right| \epsilon^{-3} d z\right) .
\end{aligned}
$$

Therefore this term goes to zero if $|\alpha| \geq 1$. (If $q<\infty$, we use Hölder inequality in the third line and get the same conclusion for all $\alpha$.)

Next we consider the term involving $\nabla \widetilde{P}$,

$$
\int_{\mathbf{R}^{3}} \nabla \widetilde{P} \epsilon^{3+|\alpha|}\left(D^{\alpha} \varphi\right)(\epsilon y) d y=-\epsilon^{3+|\alpha|+1} \int_{\mathbf{R}^{3}} \widetilde{P}\left(\operatorname{div} D^{\alpha} \varphi\right)(\epsilon y) d y .
$$

We notice that $\left(\operatorname{div} D^{\alpha} \varphi\right)(\epsilon y)$ has mean value zero and compact support. Therefore it belongs to the Hardy space $\mathcal{H}^{1}\left(\mathbf{R}^{3}\right)$ and

$$
\left\|\left(\operatorname{div} D^{\alpha} \varphi\right)(\epsilon y)\right\|_{\mathcal{H}^{1}}=\epsilon^{-3}\left\|\left(\operatorname{div} D^{\alpha} \varphi\right)(y)\right\|_{\mathcal{H}^{1}} .
$$

By the $B M O-\mathcal{H}^{1}$ pairing we have

$$
\left|\int_{\mathbf{R}^{3}} \nabla \widetilde{P} \epsilon^{3+|\alpha|}\left(D^{\alpha} \varphi\right)(\epsilon y) d y\right| \leq \epsilon^{3+|\alpha|+1}\|\widetilde{P}\|_{B M O} \cdot \epsilon^{-3}\left\|\left(\operatorname{div} D^{\alpha} \varphi\right)(y)\right\|_{\mathcal{H}^{1}} .
$$

Hence this term goes to zero for each $\alpha$.

We have shown that $F$ is some constant vector $c(c=0$ if $q<\infty)$. Hence $\nabla(\widetilde{P}-P)=c$. Therefore $(\widetilde{P}-P)(y)=(\widetilde{P}-P)(0)+c \cdot y$. The proof is complete.

Since adding a constant to $P$ does not have any effect, we can assume $P=\widetilde{P}$ in the case $2<q<\infty$. We remark that, in Section 4 , we will again define $\widetilde{P}$ by $(2.2)$, for the case when $U$ is in certain weighted $L^{p}$ space involving $A_{p}$-weights.

\section{Growth estimates: Theorem 1}

In this section we establish the growth estimates for Theorem 1 . We first derive a local gradient estimate for $U$. Then we use a bootstrap argument to obtain the polynomial growth of the pressure at infinity. Finally we use the Green's representation formula for Stokes system to improve the growth estimate of $U$. We remark that obtaining the local estimate of $\nabla U$ requires certain weak local control of $U$ and $P$. We obtain this weak control of $P$ by considering $\widetilde{P}$ given by $(2.2)$. The global control of $U$ gives us a (weak) global control of $\widetilde{P}$, which we then use to obtain a local control of $\widetilde{P}$ and $P$. It is also possible to obtain local estimates of $P$ in terms of local norms of $U$. We will discuss related estimates of the Stokes system in a forthcoming paper [ST].

In this section, we will work on balls $B=B_{\rho}\left(y_{0}\right)$ with center $y_{0} \in \mathbf{R}^{3}$ and radius $\rho<10$. 
3.1. Gradient estimate. In this subsection we prove

Lemma 3.1. Let $U$ be a weak solution of (1.3). If $U \in L^{q}, 3 \leq q<\infty$, then

$$
\|\nabla U\|_{2, B_{1}\left(y_{0}\right)}+\|U\|_{6, B_{1}\left(y_{0}\right)}=o\left(\left|y_{0}\right|^{1 / 2}\right) \quad \text { as }\left|y_{0}\right| \rightarrow \infty .
$$

Proof. We define $P$ by (2.2). For a given center $y_{0}$, let $\phi$ be a cut off function with compact support in $B_{2}\left(y_{0}\right), \phi=1$ in $B_{1}\left(y_{0}\right),|\nabla \phi|+\left|\nabla^{2} \phi\right|<20$. We take the dot product of (1.3) with $\phi U$ and then integrate. Since $U$ is smooth and $-U \cdot \Delta U=|\nabla U|^{2}-\frac{1}{2} \Delta U^{2}$, we get

$$
\begin{aligned}
& \int \nu \phi|\nabla U|^{2} d y \\
= & \int\left[\Delta \frac{\nu U^{2}}{2}-a U^{2}-(a y+U) \cdot \nabla \frac{U^{2}}{2}-\nabla P \cdot U\right] \phi d y \\
= & \int \frac{\nu U^{2}}{2} \Delta \phi-\int a U^{2} \phi+\int \frac{U^{2}}{2}(a y+U) \cdot \nabla \phi+\int 3 a \frac{U^{2}}{2} \phi+\int P U \cdot \nabla \phi .
\end{aligned}
$$

Hence

$$
\int_{B_{1}} \nu|\nabla U|^{2} d y \leq C \int_{B_{2}}\left[U^{2}+\left|y_{0}\right| U^{2}+|U|^{3}+|P U|\right] .
$$

Since $\|U\|_{3, B_{2}}$ and $\|P\|_{3 / 2, B_{2}}$ tend to zero as $y_{0}$ goes to infinity, (we recall $P$ is defined by $(2.2)$ ), we conclude that

$$
\|\nabla U\|_{2, B_{1}}=o\left(\left|y_{0}\right|^{1 / 2}\right) .
$$

By Sobolev imbedding, we get

$$
\|U\|_{6, B_{1}} \leq C\|\nabla U\|_{2, B_{1}}+C\|U\|_{q, B_{1}}=o\left(\left|y_{0}\right|^{1 / 2}\right) .
$$

Remark 3.1. If $U \in L^{\infty}$, and $\|P\|_{1, B_{1}\left(y_{0}\right)}=O\left(\left|y_{0}\right|^{N}\right)$ at infinity for some $N \geq 1$, then by (3.1) we have

$$
\|\nabla U\|_{2, B_{1}\left(y_{0}\right)}=O\left(\left|y_{0}\right|^{N / 2}\right) \quad \text { as }\left|y_{0}\right| \rightarrow \infty .
$$

3.2. Bootstrap. By iterating the Sobolev imbedding theorem and the interior $L^{p}$ estimates for Stokes system, we will derive the polynomial growth of $U$ and $P$ in this subsection. We first recall the interior $L^{p}$ estimates for Stokes system, (see [Ga I] p.208). If $(v, \pi)$ is a solution of the Stokes system

$$
\begin{array}{r}
\nu \Delta v-\nabla \pi=f \\
\operatorname{div} v=0
\end{array}
$$

in $B_{2 R}$, then

$$
\left\|\nabla^{2} v\right\|_{r, B_{R}}+\|\nabla \pi\|_{r, B_{R}} \leq C\left(\|f\|_{r, B_{2 R}}+\|v\|_{1, r, B_{2 R}-B_{R}}+\|\pi\|_{r, B_{2 R}-B_{R}}\right)
$$

for $r \in(1, \infty)$, where $C=C(\nu, r, R)$. Our situation is

$$
\begin{aligned}
\nu \Delta U-\nabla P & =F, \quad \operatorname{div} U=0 \\
F(y) & =a U+a(y \cdot \nabla) U+(U \cdot \nabla) U .
\end{aligned}
$$


For the case $3 \leq q<\infty$, we have

$$
\begin{aligned}
\|F\|_{3 / 2, B_{1}} & \leq C\left(\|U\|_{2, B_{1}}+\left|y_{0}\right|\|\nabla U\|_{2, B_{1}}+\|U\|_{6, B_{1}} \cdot\|\nabla U\|_{2, B_{1}}\right) \\
& =o\left(\left|y_{0}\right|^{3 / 2}\right)
\end{aligned}
$$

by Lemma 3.1. The interior estimate (3.2) then gives

$$
\left\|\nabla^{2} U\right\|_{3 / 2, B_{1 / 2}}+\|\nabla P\|_{3 / 2, B_{1 / 2}}=o\left(\left|y_{0}\right|^{3 / 2}\right)
$$

by Lemma 3.1 and the fact $\|P\|_{3 / 2, B_{1}}=o(1)$.

Now, using the Sobolev imbedding theorem, we get

$$
\|\nabla U\|_{3, B_{1 / 2}}+\|P\|_{3, B_{1 / 2}}=o\left(\left|y_{0}\right|^{3 / 2}\right)
$$

and hence

$$
\|U\|_{r, B_{1 / 2}}=o\left(\left|y_{0}\right|^{3 / 2}\right) \quad \text { for any } r<\infty .
$$

We now go back to $F$ and get

$$
\begin{aligned}
\|F\|_{2, B_{1 / 2}} & \leq C\left(\|U\|_{2, B_{1 / 2}}+\left|y_{0}\right|\|\nabla U\|_{2, B_{1 / 2}}+\|U\|_{6, B_{1 / 2}} \cdot\|\nabla U\|_{3, B_{1 / 2}}\right) \\
& =o\left(\left|y_{0}\right|^{2}\right) .
\end{aligned}
$$

By the interior estimate and the imbedding again:

$$
\begin{aligned}
\left\|\nabla^{2} U\right\|_{2, B_{1 / 4}}+\|\nabla P\|_{2, B_{1 / 4}} & =o\left(\left|y_{0}\right|^{2}\right) \\
\|\nabla U\|_{6, B_{1 / 4}}+\|P\|_{6, B_{1 / 4}} & =o\left(\left|y_{0}\right|^{2}\right) \\
\operatorname{osc}\left(U, B_{1 / 4}\right) & =o\left(\left|y_{0}\right|^{2}\right) .
\end{aligned}
$$

By bootstrap again, we get

$$
\operatorname{osc}\left(P, B_{1 / 8}\right)=o\left(\left|y_{0}\right|^{4}\right) .
$$

These give

$$
\left|U\left(y_{0}\right)\right|=o\left(\left|y_{0}\right|^{3}\right), \quad\left|P\left(y_{0}\right)\right|=o\left(\left|y_{0}\right|^{5}\right)
$$

Next we consider the case $q=\infty$, which requires more care. Lemma 2.1 tells us that $\widetilde{P}$ defined by $(2.2)$ is in the $B M O$ space. Hence by [JN], [FS], we have (also see [St2], p.141, 144)

$$
\begin{gathered}
\int_{\mathbf{R}^{3}}\left|\widetilde{P}(y)-\widetilde{P}_{B_{1}(0)}\right|(1+|y|)^{-3-1} d y \leq C\|\widetilde{P}\|_{B M O}, \quad \text { and } \\
\left\|\widetilde{P}-\widetilde{P}_{B_{1}\left(y_{0}\right)}\right\|_{2, B_{1}\left(y_{0}\right)} \leq C\|\widetilde{P}\|_{B M O},
\end{gathered}
$$

where $\widetilde{P}_{B_{1}\left(y_{0}\right)}$ denotes $\frac{1}{\left|B_{1}\right|} \int_{B_{1}\left(y_{0}\right)} \widetilde{P} d y$. In particular we get

$$
\|\widetilde{P}\|_{2, B_{1}\left(y_{0}\right)}=O\left(\left|y_{0}\right|^{4}\right) \text {. }
$$

Since $P-\widetilde{P}$ is affine, we conclude the same growth control for $\|P\|_{2, B_{1}\left(y_{0}\right)}$. Remark 3.1 then gives us

$$
\|\nabla U\|_{2, B_{1}\left(y_{0}\right)}=O\left(\left|y_{0}\right|^{2}\right) .
$$

We now repeat the previous bootstrap argument and end up with

$$
\left|P\left(y_{0}\right)\right|=O\left(\left|y_{0}\right|^{N}\right)
$$


for some $N>0$.

We summarize our discussion in the following

Lemma 3.2. Let $U$ be a weak solution of (1.3) and $P$ be defined as in Section 2. If $U \in L^{q}\left(\mathbf{R}^{3}\right), 3 \leq q \leq \infty$, then

$$
\left|P\left(y_{0}\right)\right|=O\left(\left|y_{0}\right|^{N}\right) \quad \text { as } y_{0} \rightarrow \infty
$$

for some $N<\infty$.

3.3. Representation formula. In this subsection we use Green's representation formula for Stokes system to get a growth control of $U$. For simplicity we will assume $\nu=1$. For the Stokes system

$$
\begin{aligned}
\Delta v-\nabla p & =f \\
\operatorname{div} v & =0
\end{aligned}
$$

in a ball $B=B_{\rho}\left(y_{0}\right)$ in $\mathbf{R}^{3}$, we have the following representation formula, (see [Ca], [Va] and [Ga I] p.234) :

$$
\begin{aligned}
v_{j}(y) & =\int_{B} G_{i j}(y, z) f_{i}(z) d z-\int_{\partial B} v_{i}(z)\left[T_{i l}\left(\mathbf{G}_{j}, g_{j}\right)(y, z)\right] n_{l}(z) d \sigma_{z}, \\
p(y) & =-\int_{B} g_{i}(y, z) f_{i}(z) d z-2 \int_{\partial B} v_{i}(z) n_{i}(z) d \sigma_{z}+\text { const. }
\end{aligned}
$$

where $T_{i l}$ denote the stress tensor

$$
T_{i l}(w, \pi)=-\delta_{i l} \pi+\left(w_{i, l}+w_{l, i}\right)
$$

and $\mathbf{G}_{j}=\left(G_{1 j}, G_{2 j}, G_{3 j}\right), G_{i j}$ and $g_{j}$ are the Green's tensors ([Ga I] p.226-227) which satisfy, for each fixed $z \in B$,

$$
\begin{aligned}
\Delta_{y} \mathbf{G}_{j}(y, z)+\nabla_{y} g_{j}(y, z) & =\delta(y-z) \mathbf{e}_{j} \\
\operatorname{div}_{y} \mathbf{G}_{j}(y, z) & =0 \\
\left.\mathbf{G}_{j}(y, z)\right|_{y \in \partial B} & =0 .
\end{aligned}
$$

Moreover, we have $G_{i j}(y, z)=G_{j i}(z, y)$, and the estimates

$$
\begin{aligned}
\left|G_{i j}(y, z)\right| & \leq c_{1}|y-z|^{-1} \\
\left|\nabla_{y} G_{i j}(y, z)\right|+\left|\nabla_{z} G_{i j}(y, z)\right|+\left|g_{j}(y, z)\right| & \leq c_{1}|y-z|^{-2}
\end{aligned}
$$

for $y, z \in \bar{B}$ (see [Ca] p.335-336). In addition, we can choose $c_{1}$ uniformly for $\rho \in\left[\frac{1}{10}, 10\right]$.

By the above estimates we have, for each fixed $y \in B$, (and we restrict $\rho \in$ $\left.\left[\frac{1}{10}, 10\right]\right)$,

$$
\begin{aligned}
& \left\|G_{i j}(y, \cdot)\right\|_{L^{s}(B)} \leq c_{2}(s) \quad \text { for } s<3, \\
& \left\|\nabla_{z} G_{i j}(y, \cdot)\right\|_{L^{s}(B)}+\left\|g_{j}(y, \cdot)\right\|_{L^{s}(B)} \leq c_{3}(s) \quad \text { for } s<3 / 2,
\end{aligned}
$$

where $c_{2}(s)$ and $c_{3}(s)$ are independent of $y \in B$.

Now we can prove

Lemma 3.3. Let $U$ be a weak solution of (1.3). If $U \in L^{q}\left(\mathbf{R}^{3}\right), 3<q<\infty$, one has

$$
U(y)=o(|y|) \quad \text { as } y \rightarrow \infty .
$$


Proof. We define $P$ by (2.2). For every ball $B=B_{\rho}\left(y_{0}\right), \rho \in[3 / 4,1]$, by the representation formula,

$$
\begin{aligned}
U_{j}(y)= & \int_{B} G_{i j}(y, z) a U_{i} d z+\int_{B} G_{i j}(y, z)(a z+U) \cdot \nabla U_{i}(z) d z \\
& -\int_{\partial B} U_{i}(z)\left[T_{i l}\left(\mathbf{G}_{j}, g_{j}\right)(y, z)\right] n_{l}(z) d \sigma_{z} .
\end{aligned}
$$

Let

$$
\begin{aligned}
I_{1} & =\int_{B} G_{i j}(y, z) a U_{i} d z \\
I_{2} & =-\int_{\partial B} U_{i}(z)\left[T_{i l}\left(\mathbf{G}_{j}, g_{j}\right)(y, z)\right] n_{l}(z) d \sigma_{z},
\end{aligned}
$$

then

$$
\begin{aligned}
U_{j}(y)= & \int_{B} G_{i j}(y, z)(a z+U) \cdot \nabla U_{i} d z+I_{1}+I_{2} \\
= & \lim _{\epsilon \rightarrow 0^{+}} \int_{B \backslash B_{\epsilon}(y)} \frac{\partial}{\partial z_{l}}\left[G_{i j}(y, z)\left(a z_{l}+U_{l}\right)\right] U_{i} d z \\
& +\int_{\partial B} G_{i j}(y, z)\left(a z_{l}+U_{l}\right) U_{i} n_{l} d \sigma_{z} \\
& -\lim _{\epsilon \rightarrow 0^{+}} \int_{\partial B_{\epsilon}(y)} G_{i j}(y, z)\left(a z_{l}+U_{l}\right) U_{i} n_{l} d \sigma_{z}+I_{1}+I_{2} .
\end{aligned}
$$

The first limit equals $I_{3}+I_{4}+3 I_{1}$ where

$$
\begin{aligned}
I_{3} & =\int_{B} \frac{\partial}{\partial z_{l}} G_{i j}(y, z) a z_{l} U_{i} d z \\
I_{4} & =\int_{B} \frac{\partial}{\partial z_{l}} G_{i j}(y, z) U_{l} U_{i} d z
\end{aligned}
$$

Let us call the middle term $I_{5}$,

$$
I_{5}=\int_{\partial B} G_{i j}(y, z)\left(a z_{l}+U_{l}\right) U_{i} n_{l} d \sigma_{z} .
$$

The second limit is zero by (3.6). Hence

$$
U_{j}(y)=4 I_{1}+I_{2}+I_{3}+I_{4}+I_{5} .
$$

If we restrict that $\left|y-y_{0}\right|<1 / 2$ and $3 / 4 \leq \rho \leq 1$, we have

$$
\begin{aligned}
& \left|I_{2}\right| \leq C c_{1} \int_{\partial B}|U| d \sigma_{z}, \\
& \left|I_{5}\right| \leq 4 c_{1} \int_{\partial B} a\left|y_{0}\right||U|+|U|^{2} d \sigma_{z} .
\end{aligned}
$$

Furthermore, since $q>3$, (hence $q^{\prime}<3 / 2$ ), we have

$$
\begin{aligned}
& \left|I_{1}\right| \leq 4 a c_{2}\left(q^{\prime}\right)\|U\|_{q, B}, \\
& \left|I_{3}\right| \leq a c_{3}\left(q^{\prime}\right)\|U\|_{q, B} \cdot\left|y_{0}\right|
\end{aligned}
$$

by Hölder inequality and (3.8), (3.9).

Finally we deal with $I_{4}$, which requires more care. If $q>6, I_{4}$ is $o(1)$ by Hölder inequality. In the case $q \in(3,6]$, if we use Hölder inequality, we will only get $I_{4}=o\left(\left|y_{0}\right|^{1+\sigma}\right)$ for any small $\sigma>0$, (the detail is left to the interested reader). 
Let us use the following weighted inequality in $R^{n}$, which is due to Leray and Hardy. For any $f \in C_{c}^{\infty}\left(R^{n}\right), 1 \leq r<n$, and any $y \in R^{n}$, we have

$$
\left\|\frac{f(z)}{|z-y|}\right\|_{r, R^{n}} \leq \frac{r}{n-r}\|\nabla f\|_{r, R^{n}} .
$$

(See [Ga I] p.59 for a proof and the references, also see [La] p.16 for the case $r=2$.) Now we set $r=2$ and choose a smooth cut-off function $\phi$ with compact support in $B_{4}(y), \phi=1$ in $B_{2}(y),|\nabla \phi|<1$. We substitute $f=\phi U$ into (3.10) and get

$$
\left\|\frac{U(z)}{|z-y|}\right\|_{2, B_{2}(y)} \leq C\|\nabla U\|_{2, B_{4}(y)}+C\|U\|_{2, B_{4}(y)}=o\left(\left|y_{0}\right|^{1 / 2}\right)
$$

by Lemma 3.1. Hence

$$
\left|I_{4}\right| \leq C \int_{B_{2}(y)} \frac{|U(z)|^{2}}{|z-y|^{2}} d z=o\left(\left|y_{0}\right|^{1}\right) .
$$

We conclude that, for $\left|y-y_{0}\right|<1 / 2$ and $\rho \in[3 / 4,1]$,

$$
\left|U_{j}(y)\right| \leq o\left(\left|y_{0}\right|^{1}\right)+C \int_{\partial B_{\rho}} a\left|y_{0}\right||U|+|U|^{2} d \sigma_{z} .
$$

Now we integrate this inequality with respect to $\rho$ from $3 / 4$ to 1 and get

$$
\begin{aligned}
|U(y)| & \leq o\left(\left|y_{0}\right|^{1}\right)+C \int_{B_{1}-B_{3 / 4}} a\left|y_{0}\right||U|+|U|^{2} d z \\
& =o\left(\left|y_{0}\right|^{1}\right) .
\end{aligned}
$$

Remark 3.2. (i) We did not use the full power of the representation formula. It is enough to assume $y=y_{0}$ in our estimates. (ii) When $q=3$, one can only show $\left|I_{3}\right|=o\left(\left|y_{0}\right|^{1+\sigma}\right)$ for any small $\sigma>0$. This is the main difficulty we will encounter if we try to use the same method to do the case $q=3$. (iii) Our analysis begins from the estimate of $\|\nabla U\|_{2, B_{1}}$, which is based on the control of $\|U\|_{3, B_{2}}$. Hence to deal with the case $q<3$ would seem to require a different idea. (iv) For $3<q<\infty$, as one easily sees, we can weaken the assumption $U \in L^{q}\left(\mathbf{R}^{3}\right)$ to

$$
\|U\|_{q, B_{2}(y)}+\|P\|_{q / 2, B_{2}(y)} \rightarrow 0 \quad \text { as }|y| \rightarrow \infty .
$$

3.4. Another approach for $3 \leq q<9$. In the case $3 \leq q<9$, one can apply the same argument of [NRS] to show

$$
|U(y)|=O\left(|y|^{-1}\right), \quad|P(y)|=O\left(|y|^{-2+\sigma}\right)
$$

for arbitrary small $\sigma>0$. The argument is as follows. We consider parabolic cylinders $Q_{1}\left(x_{0}, T\right)$ for $\left|x_{0}\right|$ large enough. We will show that the integral

$$
\int_{Q_{1}\left(x_{0}, T\right)}|u|^{3}+|p|^{3 / 2} d x d t
$$


goes to zero as $\left|x_{0}\right|$ goes to infinity. This can be done by Hölder inequality, (we recall $\left.\lambda=\lambda(t)=(2 a(T-t))^{-1 / 2}\right)$ :

$$
\begin{aligned}
\int_{Q_{1}\left(x_{0}, T\right)}|u|^{3} d x d t & =\int_{T-1}^{T} d t \int_{B_{1}\left(x_{0}\right)}|U(\lambda x)|^{3}\left(\lambda^{3} d x\right) \\
& =\int_{T-1}^{T} d t \int_{B_{\lambda}\left(\lambda x_{0}\right)}|U(y)|^{3} d y \\
& \leq \int_{T-1}^{T} d t\left(\int_{B_{\lambda}\left(\lambda x_{0}\right)}|U(y)|^{q} d y\right)^{\frac{3}{q}} \cdot C\left(\lambda^{3}\right)^{1-\frac{3}{q}} \\
& =\int_{T-1}^{T} \lambda^{3-\frac{9}{q}}(t) d t \cdot o(1) \\
& =o(1) \quad \text { if } 3 \leq q<9 .
\end{aligned}
$$

Similar estimate holds for

$$
\int_{Q_{1}\left(x_{0}, T\right)}|p|^{3 / 2} d x d t
$$

Hence by [NRS], Proposition 2.1, $u$ is bounded in $Q_{1 / 2}\left(x_{0}, T\right)$ for $\left|x_{0}\right|$ bigger than some $r_{0}$, which is independent of the direction. Therefore, by [Se], [Oh], $\nabla^{k} u$ are uniformly bounded for $|x| \in\left[r_{0}-\frac{1}{4}, r_{0}+\frac{1}{4}\right], t \in\left(T-\frac{1}{4}, T\right)$, for each $k=0,1,2 \ldots$ In terms of $U$, we get the first part of (3.11). We also need to obtain decay estimates for $P$, which is slightly more difficult than the proof of a corresponding statement in [NRS]. Nevertheless, it is possible to prove the second part of (3.11) by using the integral form of $R_{i} R_{j}$ in $\mathbf{R}^{3}$,

$$
R_{i} R_{j} f(y)=\lim _{\epsilon \rightarrow 0^{+}} \int_{|z|>\epsilon} \frac{3}{4 \pi} \frac{K(z)}{|z|^{5}} f(y-z) d z-\frac{1}{3} \delta_{i j} f(y),
$$

with $K(z)=z_{i} z_{j}-\frac{1}{3} \delta_{i j}|z|^{2}$ (cf. [St] p.73, p.58) and $f=U_{i} U_{j}$, and by estimating the above integral directly. Since we have proved Lemma 3.2 (which is enough for the proof of Theorem 1), We leave the details to the interested reader.

Another way to get (3.11) is the following. One replaces $Q_{1}\left(x_{0}, T\right)$ by $Q_{1}(0, T)$ in the previous computation and gets

$$
\int_{Q_{1}(0, T)}|u|^{3}+|p|^{3 / 2} d x d t<\infty
$$

if $3 \leq q<9$. This integral may not be small, hence we cannot use [NRS], Proposition 2.1 (which uses [CKN], Proposition 2) to show $u$ is bounded in $Q_{1 / 2}(0, T)$. Nonetheless, the proof of the same proposition shows that $(u, p)$ is a suitable weak solution in $Q_{3 / 4}(0, T)$, and therefore we can apply the results from Section 4 .

We remark that, in a recent paper by G. Tian and Z. Xin [TX], they prove the boundedness of suitable weak solutions $u$ in $Q_{R / 2}\left(x_{0}, T\right)$ under the condition

$$
\sup _{r \leq R} r^{-3} \iint_{Q_{r}(x, T)}|u(x, t)|^{2} d x d t<\epsilon_{2},
$$

where $\epsilon_{2}$ is an absolute constant. If we could apply the previous computation, we would conclude the same estimates (3.11) for $2<q<\infty$. However, [TX] assumes 
the local energy estimates (1.4) for $u$ in $Q_{R}$ which, in our case, is not a consequence of either (3.12) or the assumption $U \in L^{q}\left(\mathbf{R}^{3}\right)$.

\section{Growth estimates: Theorem 2}

In this section we establish the growth estimates for Theorem 2, that is, under the assumption of the local energy estimates (1.4) for $u$. By [CKN] p.781, (1.4) implies $\|u\|_{10 / 3, Q_{1}}<\infty$. One easily calculates that

$$
\begin{aligned}
\|u(\cdot, t)\|_{2, B_{1}} & =\lambda(t)^{-1 / 2}\|U\|_{2, B_{\lambda(t)}(0)} \\
\int_{Q_{1}}|u|^{\frac{10}{3}} d x d t & =\int_{R^{3}}|U|^{\frac{10}{3}} \cdot A_{1} \min \left(|y|^{-\frac{5}{3}}, \lambda_{0}^{-\frac{5}{3}}\right) d y, \\
\int_{Q_{1}}|\nabla u|^{2} d x d t & =\int_{R^{3}}|\nabla U|^{2} \cdot A_{2} \min \left(|y|^{-1}, \lambda_{0}^{-1}\right) d y
\end{aligned}
$$

where $\lambda_{0}=(2 a)^{-1 / 2}, A_{1}$ and $A_{2}$ are some explicit constants. Hence all the right hand sides are finite. An immediate consequence is $U \in W_{l o c}^{1,2}$. Since $u$ is a weak solution of (1.1) by assumption, $U$ is a weak solution of (1.3). Hence $U$ and $u$ are both smooth. In particular, $u$ is a "Leray-Hopf weak solution" and it differs from a "suitable weak solution" only by lacking the estimate

$$
p \in L^{5 / 4}\left(Q_{1}(0, T)\right) .
$$

From (4.1) one also has

$$
\|\nabla U\|_{2, B_{1}\left(y_{0}\right)}+\|U\|_{10 / 3, B_{1}\left(y_{0}\right)}=o\left(\left|y_{0}\right|^{1 / 2}\right) .
$$

If one assumes suitable control of $\|P\|_{3 / 2, B_{1}}$ (or certain weaker norm), one can follow the bootstrap argument in Subsection 3.2 to obtain certain polynomial growth control of $U$ and $P$. Unfortunately, the growth control of $U$ cannot be improved by using Green's representation formula because of the term $I_{3}$ in Subsection 3.3, (cf. Remark 3.2 (ii)).

Instead, we will show that $u$ is a suitable weak solution, and apply the partial regularity result from [CKN] to get the growth estimates of $U$. To show $u$ is a suitable weak solution we have to "find" a pressure $p$ satisfying (4.2). We will do this by using some weighted estimates involving $A_{p}$-weights. (See for example [St2], Chapter 5.) Since $U$ is smooth, the condition $(4.1)_{2}<\infty$ implies

$$
\int_{\mathbf{R}^{3}}|U|^{10 / 3} \cdot w(y) d y<\infty,
$$

where

$$
w(y)=|y|^{-5 / 3} .
$$

It is well known (and easily verified) that $w(y)$ is an $A_{5 / 3}$ weight in $R^{3}$, that is, the quantity

$$
F\left(y_{0}, r\right):=\left(\frac{1}{|B|} \int_{B} w(y) d y\right) \cdot\left(\frac{1}{|B|} \int_{B} w(y)^{-3 / 2} d y\right)^{2 / 3}
$$


is uniformly bounded for all balls $B=B_{r}\left(y_{0}\right)$. Using the results in [St2] p.204-211, we see that the operator $R_{i} R_{j}$ used in $(2.2)$ to define $\widetilde{P}$ is continuous on $L_{w}^{5 / 3}\left(\mathbf{R}^{3}\right)$, the space of all functions $f$ for which the norm

$$
\|f\|_{w, 5 / 3}=\left(\int_{\mathbf{R}^{3}}|f|^{5 / 3} \cdot w(y) d y\right)^{3 / 5}
$$

is finite. Therefore, given $U$ satisfying (4.3), we can set $\widetilde{P}=\sum R_{i} R_{j}\left(U_{i} U_{j}\right)$ and we have

$$
\|\widetilde{P}\|_{w, 5 / 3} \leq C \sum\left\|U_{i} U_{j}\right\|_{w, 5 / 3}
$$

Now, following the proof of Lemma 2.1 , we want to show that $\widetilde{P}$ differs from $P$ only by a constant. We first claim that $\widetilde{P}$ solves $(2.1)$ in distributional sense. This can be proved in the same way as in Lemma 2.1. The weight $w$ does not cause any new difficulty since the test-function $\varphi$ used in the proof has a compact support. By Weyl's lemma, $\widetilde{P}$ is smooth. Next we have to verify that, for the function $F(y)$ defined in Lemma 2.1, $D^{\alpha} F(0)=0$ for all $\alpha$. We consider the term involving $y \cdot \nabla U$ as an example. The computations for other terms are essentially the same.

$$
\begin{aligned}
& \left|\int_{\mathbf{R}^{3}}(y \cdot \nabla) U \epsilon^{3+|\alpha|}\left(D^{\alpha} \varphi\right)(\epsilon y) d y\right| \\
= & \left|-\epsilon^{3+|\alpha|} \int_{\mathbf{R}^{3}} 3 U\left(D^{\alpha} \varphi\right)(\epsilon y) d y-\epsilon^{3+|\alpha|} \int_{\mathbf{R}^{3}} U \epsilon y_{j}\left(\partial_{j} D^{\alpha} \varphi\right)(\epsilon y) d y\right| \\
\leq & C \epsilon^{3+|\alpha|}\left(\int_{\mathbf{R}^{3}}|U|^{10 / 3} w(y) d y\right)^{\frac{3}{10}} \cdot \\
& \cdot\left(\left.\left.\int_{\mathbf{R}^{3}}\left|\left(D^{\alpha} \varphi\right)(\epsilon y)\right| y\right|^{1 / 2}\right|^{10 / 7}+\left.\left.\left|\epsilon y_{j}\left(\partial_{j} D^{\alpha} \varphi\right)(\epsilon y)\right| y\right|^{1 / 2}\right|^{10 / 7} d y\right)^{7 / 10} \\
\leq & C \epsilon^{3+|\alpha|} \cdot\left(\int_{\mathbf{R}^{3}}\left[\left|\left(D^{\alpha} \varphi\right)(z)\right|^{\frac{10}{7}}+\left|z_{j}\left(\partial_{j} D^{\alpha} \varphi\right)(z)\right|^{\frac{10}{7}}\right]|z|^{5 / 7} \epsilon^{-5 / 7-3} d z\right)^{7 / 10} \\
= & C \epsilon^{3+|\alpha|} \cdot \epsilon^{-13 / 5} .
\end{aligned}
$$

Therefore, as $\epsilon$ goes to zero, this term goes to zero. This shows $\widetilde{P}$ together with $U$ solve Leray's equation (1.3).

Now we can define $p$ by $(1.2)_{2}$. Clearly $p$ together with $u$ solve (1.1) and $p \in$ $L^{5 / 3}\left(Q_{1}\right)$ since $\widetilde{P} \in L_{w}^{5 / 3}\left(\mathbf{R}^{3}\right)$. We summarize the above discussion in the following

Lemma 4.1. Let $u$ be a weak solution of (1.1) in $Q_{1}$ satisfying the local energy estimates (1.4). If $u$ is of the form (1.2) $)_{1}$, then $u$ is smooth, and we can find a smooth function $p \in L^{5 / 3}\left(Q_{1}\right)$ of the form (1.2) $)_{2}$ such that $(u, p)$ is a suitable weak solution of (2.1) in $Q_{1}$.

We remark that, by construction, the $L^{5 / 3}$-norm of $p$ is bounded by the local energy of $u$. More generally, by using similar arguments one can also bound $\|p\|_{r / 2, Q_{1}}$ by $\|u\|_{r, Q_{1}}^{2}$ if one assumes $u \in L^{r}\left(Q_{1}\right)$ for $r \in(2,6)$. (One uses the $A_{r / 2}$ weight $|y|^{-r / 2}$.)

We will use the following lemma, which is a variant of the Proposition 2 of [CKN]. 
Lemma 4.2. Let $(u, p)$ be a suitable weak solution of (1.1). There is an absolute constant $\epsilon_{4}>0$ such that, if

$$
\lim \sup _{r \rightarrow 0^{+}} r^{-1} \int_{Q_{r}(x, t)}|\nabla u|^{2} \leq \epsilon_{4}
$$

then $u$ is essentially bounded in $Q_{r_{1}}(x, t)$ for some $r_{1}>0$.

This lemma differs from Proposition 2 of [CKN] by replacing $Q_{r}^{*}(x, t)$ by $Q_{r}(x, t)$. (We recall that $Q_{r}^{*}(x, t)=Q_{r}\left(x, t+\frac{1}{8} r^{2}\right)$.) It assumes the information only at times previous to $t$, and gets control only at times previous to $t$. Since the original proof of [CKN], Proposition 2 and the accompanying lemmas goes through without change, we omit the details and refer the reader to [CKN]. Similar to the proof of Theorem B in [CKN], page 807, this lemma implies that the singular set at the top of the parabolic cylinder also has one-dimensional Hausdorff measure zero.

With this lemma, we can prove

Corollary 4.3. Let $(u, p)$ be a suitable weak solution of the Navier-Stokes equations (1.1) in $Q_{1}(0, T)$, and assume $u$ is of the form (1.2) $)_{1}$. Then $U(y)=O\left(|y|^{-1}\right)$.

Remark. Heuristically, if the corollary was not true, there would be a direction along which $|y||U(y)|$ is not bounded. By the self-similarity $u$ would be singular at all points on that direction (at time $T$ ), hence we have a segment consisting of singular points. We know from [CKN] that this would be impossible if they were interior points. Using Lemma 4.2, we conclude that the top of the parabolic cylinder cannot contain a singular segment either.

Proof. We may assume $T=0$. If the corollary was not true, one could find $y_{k} \in \mathbf{R}^{3}$, $\left|y_{k}\right| \rightarrow \infty$, and

$$
\left|U\left(y_{k}\right)\right| \cdot\left|y_{k}\right|>k
$$

Let us denote $y /|y|$ by $\widehat{y}$. The set $\left\{\widehat{y_{k}}\right\}$ has an accumulation point $x_{*}$ on the unit sphere $\{|y|=1\}$. We may assume that $\widehat{y_{k}} \rightarrow x_{*}$ by considering a subsequence. We claim that $u$ is not bounded in any $Q_{r}\left(\sigma x_{*}, 0\right)$ for $\sigma \in(0,1)$ and $r \in(0,1 / 2)$. To see this, let us fix $\sigma$ and $r$, and let $\lambda_{0}=\lambda\left(-r^{2}\right)=\frac{1}{\sqrt{2 a}} r^{-1}$, (we recall that $\left.\lambda(t)=(-2 a t)^{-1 / 2}\right)$. For $k$ large enough, we have $\left|y_{k}\right|>\sigma \lambda_{0}$ and $\left|\sigma \widehat{y_{k}}-\sigma x_{*}\right|<r$. Let $t_{k}$ be the time which satisfies $\lambda\left(t_{k}\right) \cdot \sigma=\left|y_{k}\right|$. One checks easily that $\lambda\left(t_{k}\right)=$ $\sigma^{-1}\left|y_{k}\right|>\lambda_{0}$ and hence $t_{k} \in\left(-r^{2}, 0\right)$. Therefore the point $\left(\sigma \widehat{y_{k}}, t_{k}\right)$ is contained in $Q_{r}\left(\sigma x_{*}, 0\right)$. On the other hand,

$$
\left|u\left(\sigma \widehat{y_{k}}, t_{k}\right)\right|=\lambda\left(t_{k}\right) \cdot\left|U\left(\lambda\left(t_{k}\right) \sigma \widehat{y_{k}}\right)\right|=\sigma^{-1}\left|y_{k}\right| \cdot\left|U\left(y_{k}\right)\right|>\sigma^{-1} k .
$$

This shows the claim, that is, all points on the segment $\left\{\left(\sigma x_{*}, 0\right): \sigma \in(0,1)\right\}$ are singular. This is a contradiction to the fact that the singular set at the top of the parabolic cylinder has one-dimensional Hausdorff measure zero. This contradiction shows our corollary.

To finish the proof of Theorem 2, we have several possibilities. The first way is to observe that, since $U$ is smooth, $U \in L^{q}\left(\mathbf{R}^{3}\right)$ for $q>3$ by Lemma 4.1 and Corollary 4.3. Therefore Theorem 2 follows from Theorem 1 . The second way is to prove

$$
P(y)=o\left(|y|^{N}\right) \quad \text { as } y \rightarrow \infty
$$


for some $N<\infty$. (Corollary 4.3 and (4.4), together with Lemma 5.1 in next section, will prove Theorem 2 without using Theorem 1.) To prove (4.4), one can use Lemma 4.1 and follow the proof of Subsections 3.1 and 3.2, as sketched at the beginning of this section. Alternatively, since Corollary 4.3 implies that $u$ is uniformly bounded in $\left\{x: \frac{1}{4}<|x|<1\right\} \times(-1,0)$, the same argument of Subsection 3.4 gives the second part of (3.11).

\section{The Liouville-type lemma and the main theorems}

In this last section we prove the key Liouville type lemma and the main theorems.

Lemma 5.1. Let $\Pi: \mathbf{R}^{3} \rightarrow \mathbf{R}$, and $U: \mathbf{R}^{3} \rightarrow \mathbf{R}^{3}$ be smooth, and satisfy

$$
-\nu \Delta \Pi(y)+(U(y)+a y) \cdot \nabla \Pi(y) \leq 0
$$

in $\mathbf{R}^{3}$. If for some constant $b \in(0, a)$ we have $|U(y)| \leq b|y|$ for $|y|$ sufficiently large, and

$$
|\Pi(y)|=o\left(\int^{|y|} e^{c s^{2} / 2} d s\right) \quad \text { as }|y| \rightarrow \infty,
$$

where $c=(a-b) / \nu$, then $\Pi$ is constant.

Proof. Let $M(r)=\max _{|y|=r} \Pi(y) . M(r)$ is non-decreasing in $r$ by maximal principle. Let

$$
\phi(r)=\frac{-1}{c r} e^{c r^{2} / 2}+\int^{r} e^{c s^{2} / 2} d s .
$$

(It solves (1.12) up to a constant factor.) It is easy to see that the function $v(y)=$ $\phi(|y|)$ is a super solution for $|y|$ bigger than some $r_{0}$, i.e.,

$$
-\nu \Delta v(y)+(U(y)+a y) \cdot \nabla v(y) \geq 0 \quad \text { for }|y|>r_{0} .
$$

Let

$$
\psi_{\epsilon}(y)=M\left(r_{0}\right)+\epsilon \cdot\left[\phi(|y|)-\phi\left(r_{0}\right)\right] \quad \text { for } \epsilon>0 .
$$

Then $\psi_{\epsilon}$ are all super solutions for $|y|>r_{0}$. It is clear that $\psi_{\epsilon}(y) \geq \Pi(y)$ for $|y|=r_{0}$ and for $|y|$ near $\infty$ by the growth of $\Pi$. By comparison principle we have

$$
\Pi(y) \leq M\left(r_{0}\right)+\epsilon \cdot\left[\phi(|y|)-\phi\left(r_{0}\right)\right] \quad \text { for }|y| \geq r_{0} .
$$

Now letting $\epsilon$ go to zero, we get $\Pi(y) \leq M\left(r_{0}\right)$ for all $|y| \geq r_{0}$, and hence max $\Pi$ is attended at some $y,|y|=r_{0}$. By the strong maximal principle $\Pi$ must be constant. (Notice that our coefficients are bounded in bounded regions.)

Remark 5.1. It is reasonable to expect that $(U+a y) \cdot \nabla \Pi$ acts as a "magnifying force", since we are looking for blow-up solutions of the Navier-Stokes equations, (cf. [GK] equation (3.2) and [Gi2]). It is known that for Navier-Stokes equations there exist forward self-similar solutions which are defined on $\mathbf{R}^{3} \times(0, \infty)$ and are singular at $(0,0)$, (see $[\mathrm{GM}],[\mathrm{CP}]$ ). Its corresponding stationary problem behaves like $\Delta v(y)=-y \cdot \nabla v(y)$ at infinity, which does not blow up at infinity. 
Remark 5.2. One can easily extend this lemma to $\mathbf{R}^{n}, n \geq 2$. In that case, the comparison function $\phi$ takes the form

$$
\phi(r)=\int^{r} s^{1-n} e^{c s^{2} / 2} d s
$$

Now we prove Theorem 1 and Theorem 2 .

Proof of Theorem 1 and Theorem 2. Lemmas 3.2 and 3.3, respectively Corollary 4.3 and the estimate (4.4), give us the growth estimates

$$
U(y)=o(|y|), \Pi(y)=O\left(|y|^{N}\right) \quad \text { as }|y| \rightarrow \infty
$$

under either Theorem 1 or Theorem 2, for some $N<\infty$. Lemma 5.1 then implies that $\Pi$ is constant. Therefore $\nabla \Pi$ is zero, i.e.,

$$
U_{j} U_{j, i}+P_{, i}+a U_{i}+a y_{j} U_{j, i}=0 \quad \text { for each } i
$$

Besides (5.2), if we consider (1.7), we get $|\Omega(y)|^{2}=0$, that is

$$
\partial_{i} U_{j}=\partial_{j} U_{i} \quad \text { for all } i, j \text {. }
$$

Comparing (5.2) with the equations (1.3) of $U$

$$
-\nu \Delta U_{i}+U_{j} U_{i, j}+P_{, i}+a U_{i}+a y_{j} U_{i, j}=0 \quad \text { for each } i,
$$

we get

$$
-\nu \Delta U_{i}=0 \quad \text { for each } i \text {. }
$$

Since $U \in L^{q}\left(\mathbf{R}^{3}\right)$ in Theorem 1 and $U \rightarrow 0$ at infinity in Theorem 2, the usual Liouville theorem implies $U_{i}$ are 0 , (or constant if $U \in L^{\infty}$ ). The proof is complete.

Remark 5.3. By Lemma 5.1, it is sufficient to have $|U(y)| \leq b|y|$ for some $b<a$ and for $|y|>r_{0}$, (and $P(y)=O\left(|y|^{N}\right)$ for some $N>0$ ), for the triviality of $U$. The corresponding condition for $u$ (given by $(1.2)_{1}$ ) is

$$
|u(x, t)| \leq \frac{b|x|}{2 a(T-t)} \quad \text { for }|x|>(T-t)^{\frac{1}{2}} r_{0} .
$$

It is interesting to compare it with (1.6).

Remark 5.4. To conclude that a solution of (1.3) is zero, certain assumptions on the growth of $U$ are necessary, as can be seen from the following example. Let $\Phi$ be an arbitrary harmonic function on $\mathbf{R}^{3}$. Let $U=\nabla \Phi$ and $P=-\frac{1}{2}|U|^{2}-$ $a y \cdot U$, (i.e. $\Pi=0$ ). Then $(U, P)$ solves Leray's equations (1.3). This gives us certain heuristic reason for considering the quantity $\Pi$. The fact that the quantity $\frac{1}{2}|u|^{2}+p$ satisfies a maximal principle for the stationary Navier-Stokes equations is well-known (see e.g. [Se2, p.261], [GW]), and has played an important role in recent results ([FR1,2], [Str3]) regarding the regularity of solutions of the stationary Navier-Stokes equations in higher dimensions.

Remark 5.5. Scheffer [Sch2] raised the question of the existence of nontrivial solutions of Leray's equation with a "speed-reducing" force $g$ :

$$
-\nu \Delta U+a U+a y \cdot \nabla U+U \cdot \nabla U+\nabla P=g, \quad \operatorname{div} U=0
$$


for some $U, g$ with $U \cdot g \leq 0$. By using the methods in this paper we can obtain some partial results on Scheffer's question, but the general case seems to remain open.

Acknowledgment. The author wishes to express his deep gratitude to his graduate advisor, Professor Vladimír Šverák, for his guidance and encouragement. He also wishes to thank Professor R. V. Kohn for helpful comments, and his friends Yu Yuan, Y.-P. Kuo, Tim Brule and S.-C. Chu for continual interests in this work. This work was partially supported by NSF grant DMS-9622795.

\section{REFERENCES}

[CKN] L. Caffarelli, R. V. Kohn, L. Nirenberg, "Partial regularity of suitable weak solution of the Navier-Stokes equations", Comm. Pure Appl. Math. 35 (1982), 771-831.

[CZ] A. P. Caldreón, A. Zygmund, "On existence of certain singular integrals", Acta Math. 88 (1952), 85-139.

[CP] M. Cannone, F. Planchon, "Self-similar solutions for Navier-Stokes equations in $\mathbf{R}^{3}$ ", Comm. in P.D.E. 21(1\&2) (1996), 179-193.

[Ca] L. Cattabriga, "Su un problema al retorno relativo al sistema di equazioni di Stokes", Rend. Sem. Math. Univ. Padova 31 (1961), 308-340.

[CF] P. Constantin, C. Fefferman, "Direction of vorticity and the problem of global regularity for the Navier-Stokes equations", Indiana Univ. Math. J. 42 (1993), no. 3, 775-789.

[CFo] P. Constantin, C. Foias, "Navier-Stokes equations", Univ. of Chicago Press, 1988.

[FJR] E. B. Fabes, B. F. Jones, N. M. Riviere, "The initial value problem for the Navier-Stokes equations with data in $L^{p}$ ", Arch. Rat. Mech. Anal. 45 (1972), 222-240.

[FS] C. Fefferman, E. Stein, " $\mathcal{H}^{p}$ spaces of several variables", Acta Math. 129 (1972), 137-193.

[FR1] J. Frehse, M. Růžička, "On the regularity of the stationary Navier-Stokes equations", Ann. Scu. Norm. Pisa 21 (1994), 63-95.

[FR2] J. Frehse, M. Růžičča, "Regularity of the stationary Navier-Stokes equations in bounded domains", Arch. Rat. Mech. Anal. 128 (1994), 361-381.

[Ga] G. Galdi, "An introduction to the mathematical theory of Navier-Stokes equations", I, II, Springer-Verlag, 1994.

[GiM] M. Giaquinta, G. Modica, "Non linear systems of the type of the stationary Navier-Stokes equations", J. Reine Angew. Math. 330 (1982), 173-214.

[Gi] Y. Giga, "Solutions for semilinear parabolic equations in $L^{p}$ and regularity of weak solutions of the Navier-Stokes system", J. Diff. Equations 61 (1986), 186-212.

[Gi2] Y. Giga, "On elliptic equations related to self-similar solutions for nonlinear heat equations", Hiroshima Math. J. 16 (1986), 539-552.

[GK] Y. Giga, R. V. Kohn, "Asymptotically self-similar blow-up of semilinear heat equations", Comm. Pure Appl. Math. 38 (1985), 297-319.

[GM] Y. Giga, T. Miyakawa, "Navier-Stokes flow in $\mathbf{R}^{3}$ with measures as initial vorticity and Morrey spaces", Comm. in P.D.E. 14(5) (1989), 577-618.

[GW] D. Gilbarg, H. F. Weinberger, "Asymptotic properties of Leray's solution of the stationary two-dimensional Navier-Stokes equations", Russian Math. Surveys, 29:2 (1974), 109-123.

[HW] John G. Heywood, Owen D. Walsh, "A counterexample concerning the pressure in the Navier-Stokes equations, as $t \rightarrow 0^{+}$", Pacific J. Math. 164 (1994), no. 2, 351-359.

[Ho] E. Hopf, "Über die Anfangswertaufgabe für die hydrodynamischen Grundgleichungen", Math. Nachr. 4 (1951), 213-231.

[JN] F. John, L. Nirenberg, "On functions of bounded mean oscillation", Comm. Pure Appl. Math. 14 (1961), 415-426.

[KL] N. Kopell, M. Landman, "Spatial structure of the focusing singularity of the nonlinear Schrödinger equation: a geometrical analysis", SIAM. J. Appl. Math. 55 (1995), no. 5, 1297-1323. 
[La] O. A. Ladyzhenskaya, "The mathematical theory of viscous incompressible flow", 2nd ed., Gordon and Breach, 1969.

[Le] J. Leray, "Sur le mouvement d'un liquide visqueux emplissant l'espace", Acta Math. 63 (1934), 193-248.

[LL] F.H. Lin, C. Liu, "Partial regularity of the dynamic system modeling the flow of liquid crystals", Discrete Contin. Dynam. System 2 (1996), no 1, 1-22.

[NRS] J. Nečas, M. Růžička, V. Šverák, "On Leray's self-similar solutions of the Navier-Stokes equations", Acta Math. 176 (1996), 283-294.

[MNPS] J. Málek, J. Nečas, M. Pokorný, M.E. Schonbek, "On possible singular solutions to the Navier-Stokes equations", preprint.

[Oh] T. Ohyama, "Interior regularity of weak solutions to the Navier-Stokes equations", Proc. Japan Acad. 36 (1960), 273-277.

[Sch1] V. Scheffer, "Hausdorff measure and the Navier-Stokes equations", Comm. Math. Phys. 55 (1977), no. 2, 97-112.

[Sch2] V. Scheffer, "A solution to the Navier-Stokes inequality with an internal singularity", Comm. Math. Phys. 101 (1985), no. 1, 47-85.

[Se] J. Serrin, "On the interior regularity of weak solutions of the Navier-Stokes equations", Arch. Rat. Mech. Anal. 9 (1962), 187-195.

[Se2] J. Serrin, "Mathematical principles of classical fluid mechanics", in Handbuch der Physik, 8:1, pp. 125-263, Springer-Verlag, Berlin-Göttingen-Heidelberg, 1959.

[SvW] H. Sohr, W. von Wahl, "On the regularity of the pressure of weak solutions of NavierStokes Equations", Arch. Math. 46 (1986), 428-439.

[St] E. M. Stein, "Singular integrals and the differentiability properties of functions", Princeton University Press, 1970.

[St2] E. M. Stein, "Harmonic analysis: real-variable methods, orthogonality, and oscillatory integrals", Princeton University Press, 1993.

[Str] M. Struwe, "On the evolution of harmonic maps in higher dimensions", J. Diff. Geom. 28 (1988), 485-502.

[Str2] M. Struwe, "On partial regularity results for the Navier-Stokes equations", Comm. Pure Appl. Math. 41 (1988), 437-458.

[Str3] M. Struwe, "Regular solutions of the stationary Navier-Stokes equations on $\mathbf{R}^{5}$ ", Math. Ann. 302 (1995), 719-741.

[ST] V. Šverák, T.-P. Tsai, to appear.

[Ta] S. Takahashi, "On interior regularity criteria for weak solutions of the Navier-Stokes equations", Manuscripta Math. 69 (1990), no. 3, 237-254.

[Te] R. Temam, "Navier-Stokes equations: theory and numerical analysis", North-Holland, Amsterdam and New York, 1977.

[TX] G. Tian, Z. Xin, "Gradient estimation on Navier-Stokes equations", preprint.

[Va] A. Valli, "On the integral representation of the solution to the Stokes system", Rend. Sem. Mat. Univ. Padova 74 (1985), 85-114.

[vW] W. von Wahl, "The equations of Navier-Stokes and abstract parabolic equations", Vieweg, 1985.

School of Mathematics, University of Minnesota, Minneapolis, MN 55455, USA

E-mail address: ttsai@math.umn.edu 